\title{
Vie et mort d'une souris Huntington
}

«La roche Tarpéienne est proche du Capitole.» C'est sans doute l'adage qui a dû revenir en mémoire à Gillian Bates (Guy's Hospital, Londres, G.-B.) après les révélations dévastatrices de deux équipes américaines sur sa «souris Huntington» qui, jusque-là, avait fait d'elle l'une des scientifiques les plus couvertes de louanges, d'invitations et de prix de ces deux dernières années. Autant le tapage qui a entouré cette souris était déplacé, autant il faut garder la tête froide à l'heure du bilan pour ne pas jeter les bébés qu'elle a fait naître avec l'eau du bain.

La souris (dite «lignée R6/2»), avait été construite par l'équipe de Bates en 1996 en introduisant par microinjection un fragment du gène codant pour la huntingtine, provenant d'un patient, qui comprenait à peu près $1 \mathrm{~kb}$ de séquence non traduite en 5', l'exon 1 contenant une très importante répétition de triplets CAG (plus de 140 , ce qui est très audelà du nombre observé chez la quasi-totalité des patients) et les premières 262 bp du premier intron [1]. Le phénotype observé était très complexe mais impliquait, à partir de la $9^{\text {e }}$ semaine, une atteinte neurologique, la souris décédant 1 à 4 semaines après le début des troubles. Les symptomes neurologiques comprenaient un tremblement de repos, des mouvements stéréotypés et une ataxie, ainsi que, de temps en temps, des mouvements involontaires du tronc. Il existait également des crises d'épilepsie sévères, pouvant durer plusieurs minutes. Ce sont ces symptômes, largement vantés comme "très comparables" à ceux présentés par les patients, qui ont fait la réputation du modèle même si, devant des différences majeures évidentes (le tremblement ou les crises d'épilepsie ne font pas partie de la clinique humaine), un rapprochement était fait plutôt avec les (rares) formes juvéniles dites «syndrome de Westphal». Il existait, toutefois, un très gros problème dès cette époque, celui de l'absence totale d'atteinte neuronale visible dans le striatum, siège d'un dysfonctionnement neuronal précoce, et d'une dégénérescence progressive massive chez les malades. Le cerveau des souris à l'autopsie était globalement plus petit (de 20\%) que celui de leurs congénères non transgéniques, mais il s'agissait d'une réduction globale, sans signe de localisation ou de gliose, et elle co-existait avec une perte relative de poids corporel comparable.

Pourquoi ces souris mourraient-elles, c'est la question que se sont posés, deux ans plus tard, les membres de l'équipe de Curt Freed (Denver, CO, USA) confrontés à l'échec total d'expériences destinées à évaluer l'effet de greffes de neurones fotaux dans le striatum de ces souris [2] (échec confirmé depuis par une équipe anglaise associée à celle de Bates [3]). Présentés au colloque de la Society for Neuroscience de Los Angeles le 9 novembre, sans que l'abstract en ait révélé la teneur, les résultats de l'équipe de Freed étaient aussi secs que cruels: glycémie au plafond, insuline au plancher... la souris «Huntington» était mortellement diabétique! Ce diagnostic collait parfaitement, d'ailleurs, avec la polyurie massive et la perte de poids sans hypophagie des souris, déjà soulignées par l'équipe de Bates [1]. Flynt Beal (Harvard, MA, USA) avait atteint les mêmes conclusions après une étude par spectroscopie du glucose cérébral en résonance magnétique nucléaire mais ne les présenta pas dans la communication sur cette technique qu'il co-signait avec Bates à Los Angeles [4]. Il le fit, 10 jours plus tard, dans le cadre beaucoup plus familial du colloque du Huntington Study Group à Palm Beach dans lequel, de toute façon, tout le monde ne parlait plus que de cela. Les conséquences neurologiques du diabète étant, comme chacun le sait, considérables, le glas sonnait pour le phénotype neurologique «Huntington » de la lignée R6/2.

L'aspect un peu théâtral qu'a eu la chute très brutale de cette souris considérée pendant deux ans comme le modèle - génotypique et phénotypique - de la maladie de Huntington a été à la mesure de l'engouement excessif qui l'avait entourée jusqu'alors. Une des associations américaines de patients Huntington en avait ainsi fait «la découverte la plus importante depuis l'identification de la maladie» (qui date de 1872 !). Il ne faudrait pas que, par un retour de balancier tout aussi peu raisonné, le bilan de deux années de travail sur ce modèle soit aujourd'hui brutalement éliminé. C'est en effet grâce à l'étude de cette souris R6/2 qu'un groupe collaboratif anglo-américain coordonné par Bates a mis en évidence l'an dernier des inclusions intranucléaires dans les neurones, notamment striataux et corticaux [5], qui ont immédiatement été retrouvées dans le cerveau de patients décédés des suites d'une maladie de Huntington [6]. Frédéric Saudou, dans l'équipe de Greenberg 
(Harvard, MA, USA), a certes très récemment modulé un peu le message convoyé jusque-là, qui faisait de ces inclusions la cause de la dégénérescence neuronale [7], et plusieurs autres travaux rapportés par Marian DiFiglia au colloque de la Société des Neurosciences lors d'un symposium sur l'utilisation des souris transgéniques pour l'étude des maladies neurodégénératives [8] ou très récemment publiés [9] confirment l'absence de liaison stricte entre les deux phénomènes. Il n'en reste pas moins que les inclusions intranucléaires neuronales forment un aspect particulièrement intéressant de toutes les maladies génétiques associées à l'expansion d'une répétition de triplets CAG [10]. En tout état de cause, l'aspect qui restera le plus positif de toute cette aventure est sans conteste l'intense recherche que cette souris a stimulée sur elle, mais aussi, d'une certaine façon, en compétition avec elle. Les nouvelles "souris Huntington", aujourd'hui construites par introduction $\mathrm{du}$ gène ou de l'ADNc de la Huntingtine complets commencent à pointer leur nez [11, 12], et on peut sans doute en attendre encore beaucoup pour la compréhension de la physiopathologie de la maladie de Huntington et de ses voisines.

M.P.

1. Mangiarini L, Sathasivam K, Seller M, et al. Exon 1 of the HD gene with an expanded CAG repeat is sufficient to cause a progressive neurological phenotype in transgenic mice. Cell 1996; 87: 493-506.

2. Hurlbert MS, Kaddis FG, Zhou W, Bell KP, Hutt CJ, Freed CR. Neural transplantation in a transgenic mouse model of Huntington's disease. Neuroscience Abst 1998; 24: 380-9.

3. Dunnett SB, Carter RJ, Watts C, Torres EM, Mahal A, Mangiarini L, Bates G, Morton AJ. Striatal transplantation in a transgenic mouse model of Huntington's disease. Exp Neurol 1998; 154: 3140.

4. Browne SE, Berger SC, Mangiarini L, Mahal A, Bates GP, Beal MF. Cerebral energy metabolism in huntingtin mutant R6/2 transgenic mice. Neuroscience Abst 1998; 24: 599-1.

5. Davies SW, Turmaine M, Cozens BA, DiFiglia M, Sharp AH, Ross CA, Scherzinger E, Wanker
EE, Mangiarini L, Bates GP. Formation of neuronal intranuclear inclusions undierlies neurological dysfunction in mice transgenic for the HD mutation. Cell 1997; 90 : 537-48.

6. DiFiglia M, Sapp E, Chase KO, Davies SW, Bates GP, Vonsattel JP, Aronin N. Science 1997; 277: 1990-3.

7. Saudou F, Finkbeiner S, Devys D, Greenberg ME. Huntingtin acts in the nucleus to induce apoptosis but death does not correlate with the formation of intranuclear inclusions. Cell 1998; 95: 55-66.

8. DiFiglia M, in Neurodegenerative diseases: lessons from transgenic models. Neuroscience Abst $1998 ; 24: 785$.

9. Martindale D, Hackam A, Wieczorek A, Ellerby L, Wellington C, McCutcheon K, Singaraja R, Kazemi P, Devon R, Kim SU, Bredesen DE, Tufaro F, Hayden MR. Length of huntingtin and its polyglutamine tract influences localization and frequency of intracellular aggregates. Nat Gen $1998 ; 18: 150-4$.

10. Kim TW, Tanzi RE. Neuronal intranuclear inclusions in polyglutamine diseases: nuclear weapons or nuclear fallout? Neuron 1998; 21 : 657-9.

11. Reddy PH, Williams M, Charles V, Garrett L, Pike-Buchanan L, Whetsell WO, Miller G, Tagle DA. Behavioural abnormalities and selective neuronal loss in HD transgenic mice expressing mutated full-length HD cDNA. Nat Gen 1998; 20: 198-202.

12. Hodgson G, Agoyan N, Smith D, et al. YAC transgenic mice expressing mutant human huntingtin show deficits in hippocampal long-term potentiation. Neuroscience Abst 1998; 24: 127-1.

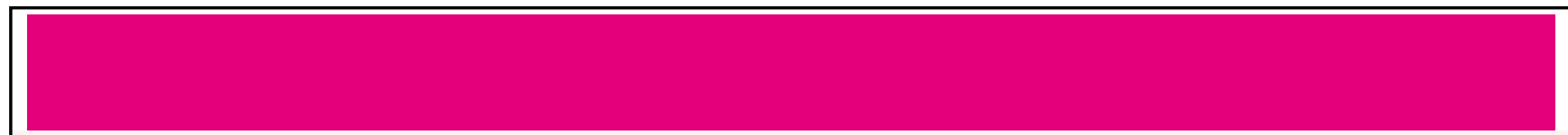

